\title{
Urgences
}

\section{La petite géante...}

\section{Nicole Bonin}

Numéro 8, 4e trimestre 1983

Littérature jeunesse

URI : https://id.erudit.org/iderudit/025120ar

DOI : https://doi.org/10.7202/025120ar

Aller au sommaire du numéro

Éditeur(s)

Urgences

ISSN

0226-9554 (imprimé)

1927-3924 (numérique)

Découvrir la revue

Citer ce document

Bonin, N. (1983). La petite géante... Urgences, (8), 53-59.

https://doi.org/10.7202/025120ar

Ce document est protégé par la loi sur le droit d'auteur. L'utilisation des services d'Érudit (y compris la reproduction) est assujettie à sa politique d'utilisation que vous pouvez consulter en ligne.

https://apropos.erudit.org/fr/usagers/politique-dutilisation/
Cet article est diffusé et préservé par Érudit.

Érudit est un consortium interuniversitaire sans but lucratif composé de l’Université de Montréal, l'Université Laval et l'Université du Québec à Montréal. Il a pour mission la promotion et la valorisation de la recherche. https://www.erudit.org/fr/ 


\section{NICOLE BONIN}

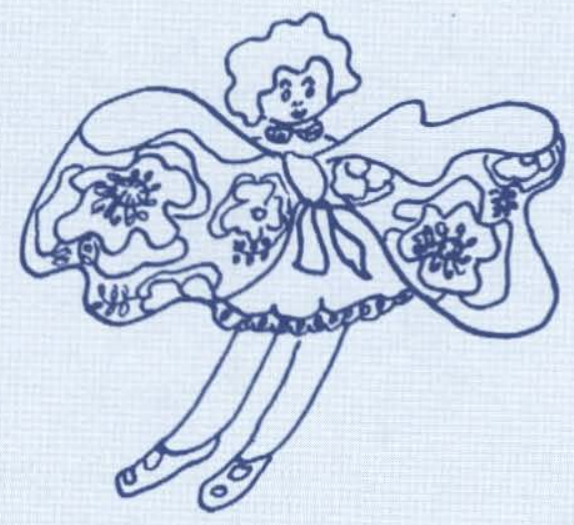




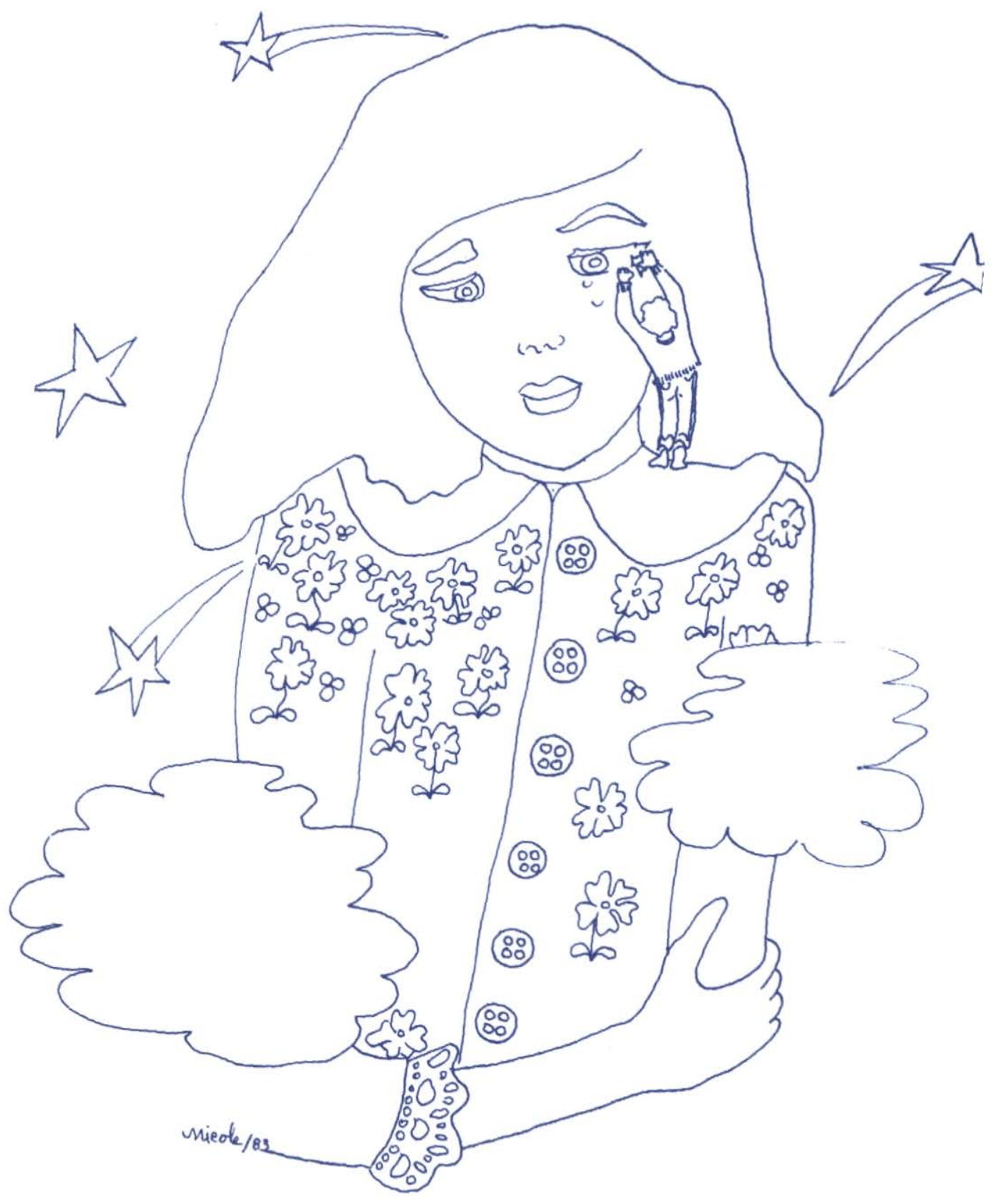




\section{la petite géante...}

Nicolas aVAit erimpe ausSi haut que possible, MAIS IL ENTENDAIT TOUJOURS CE LEGER BRUIT DE SANGLOT QUI ANAIT ATIIRE SON ATTENTION. LORSQU'IL EOUT ENFIN DÉPASSÉ LES NUAGES, IL APERCUT UNE GRANDE PETITE FILLE, UNE PETITE fILLE GÉANTE, QUI PLEURAIT, TOUTE SEULE. SUR LE HAUT DE LA MONTAGNE.

-" ohe! pourquoi pleures-tu?". cria nicolas en METTANT SES MAINS EN PORTE-VOIX. LA PETITE FILLE CESSA UN MOMENT DE SANGLOTER ET REGARDA NICOLAS.

- Je pleure marce que j'al quelque chose dans l'oeil, ET QUE CA FAIT MAL, ET QUE MON PAPA M'A DIT DE RESTER LA. QU'iL ALLAiT REVENIR... "RE'PONDIT-ELLE ENFIN, TOUT D'UNE TRAITE. APRES QUOI. ELLE SE REMIT A PLEURER DE PLUS BELLE.

- "aie! mes oreilles ! ce qu'elle fait du bruit !- Se Dit NICOLAS. "GRIMPE-MOI SUR TON ÉPAULE, PEUT-ÊTRE QUE JE TOURRAI T'AIDER...

alors, La grande petite fillLe ramassa délicatement NICOLAS ET LE POSA SUR SON ÉPAOLE. ET NICOLAS DE SE HAUSSER SUR LA POINTE DES PIEDS POUR ATTEINDRE L'OEIL EMBARRASSÉ.

-" ne ferme pas les yeux!" lui dit-il en évitant de JUSTESSE DE SE COINCER LEPETTT DOIGT SOUS LA PAUPIÈrE GÉANTE.

NICOLAS EÜT BEAUCOUP DE PEINE A SOULEVER CE QUI LUI SEMBLAIT ĖTRE UNE ÉNORME PIERRE, INSTALLĖE LA. DANS LE COIN DE L'OEIL. MAIS iL Y RÉUSSIT ENFIN

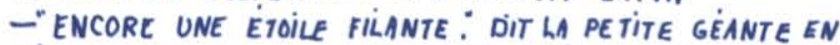
SÉCHANT SES larmes. "Au reVOir. Nicolas et merci!: Fit. ELLE EN LE dÉPOSANT PAR TERRE. ET ELLE S'EN ALLA, COURANT A TOUTES JAMBES, ECRASANT SOUS SES PAS D'ÉNOR. MES ÉPINETTES.

- Quelle géante désobeissante ! son pere lui avait dit DE L'ATIENDRE... - PENSAitT NICOLAS EN RETOURNANT CHEZ LUI. ET iL SE DÉPECHA. CAR SA MAMAN LUI AVAIT DIT DE RESTER TOUT RRES DE LA MAISON. 


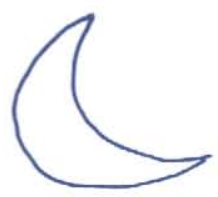

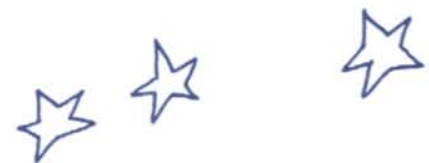

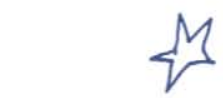

12

(5)

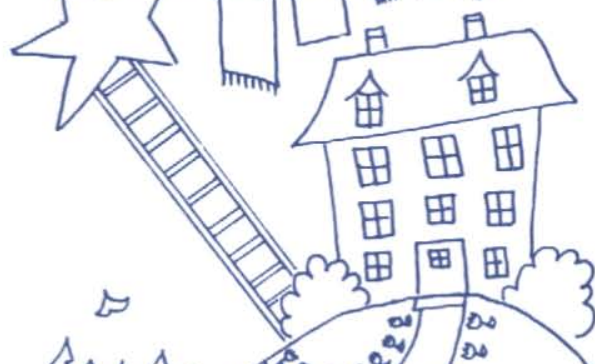

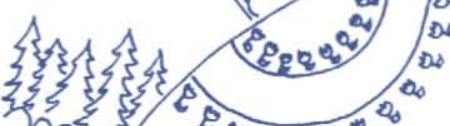

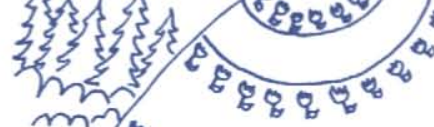
悲等

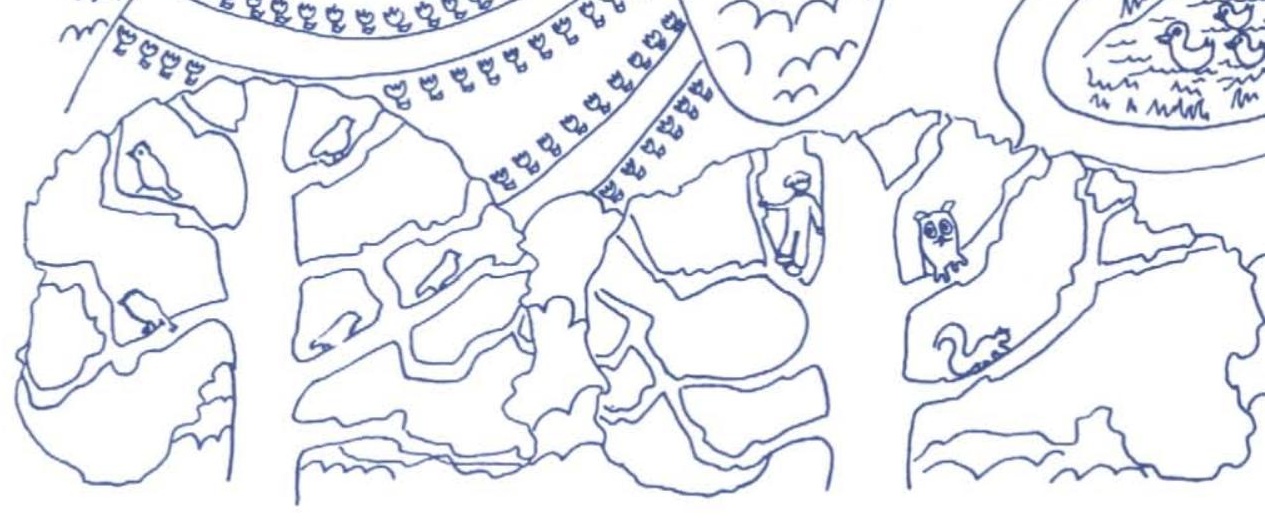




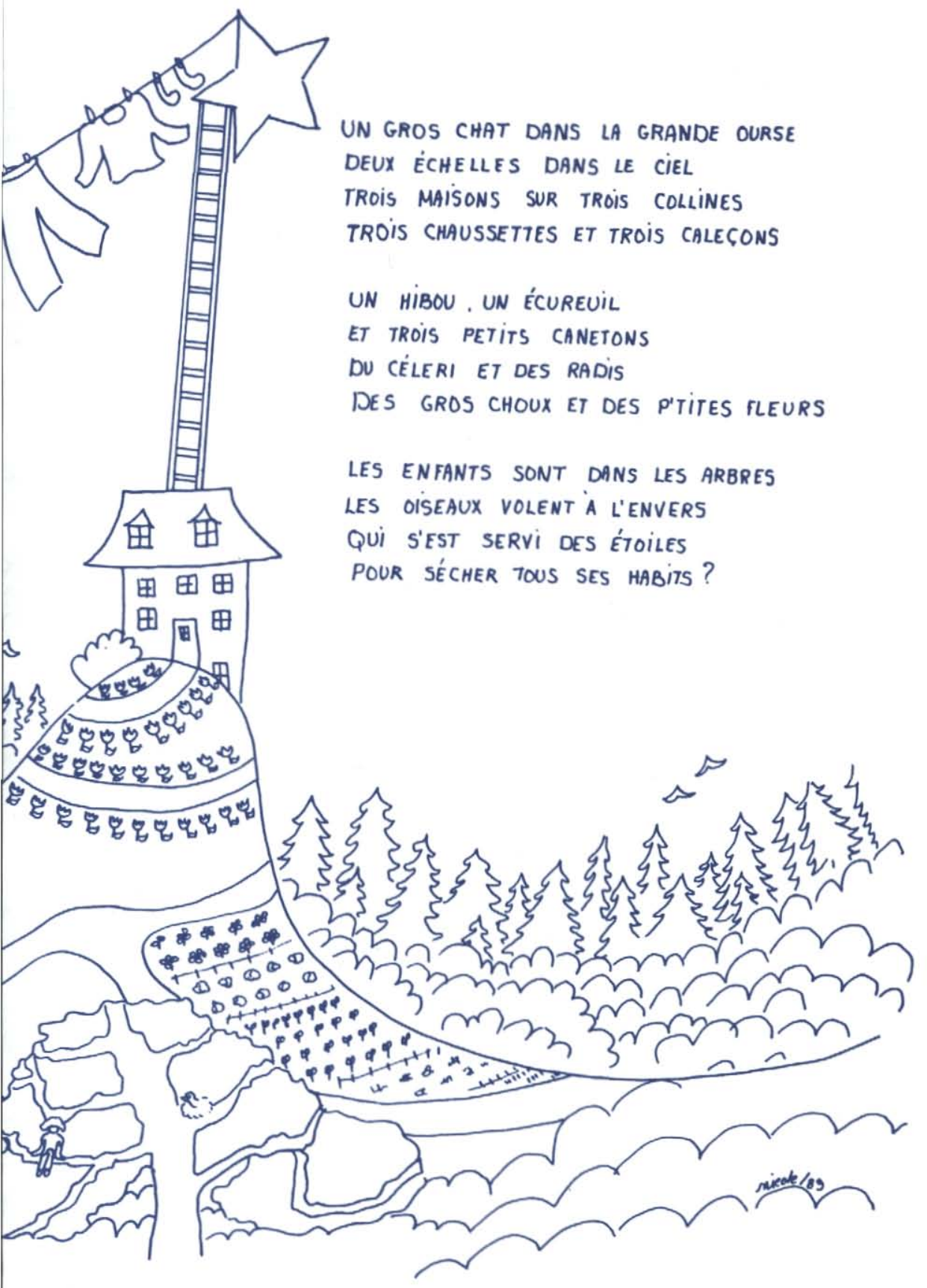




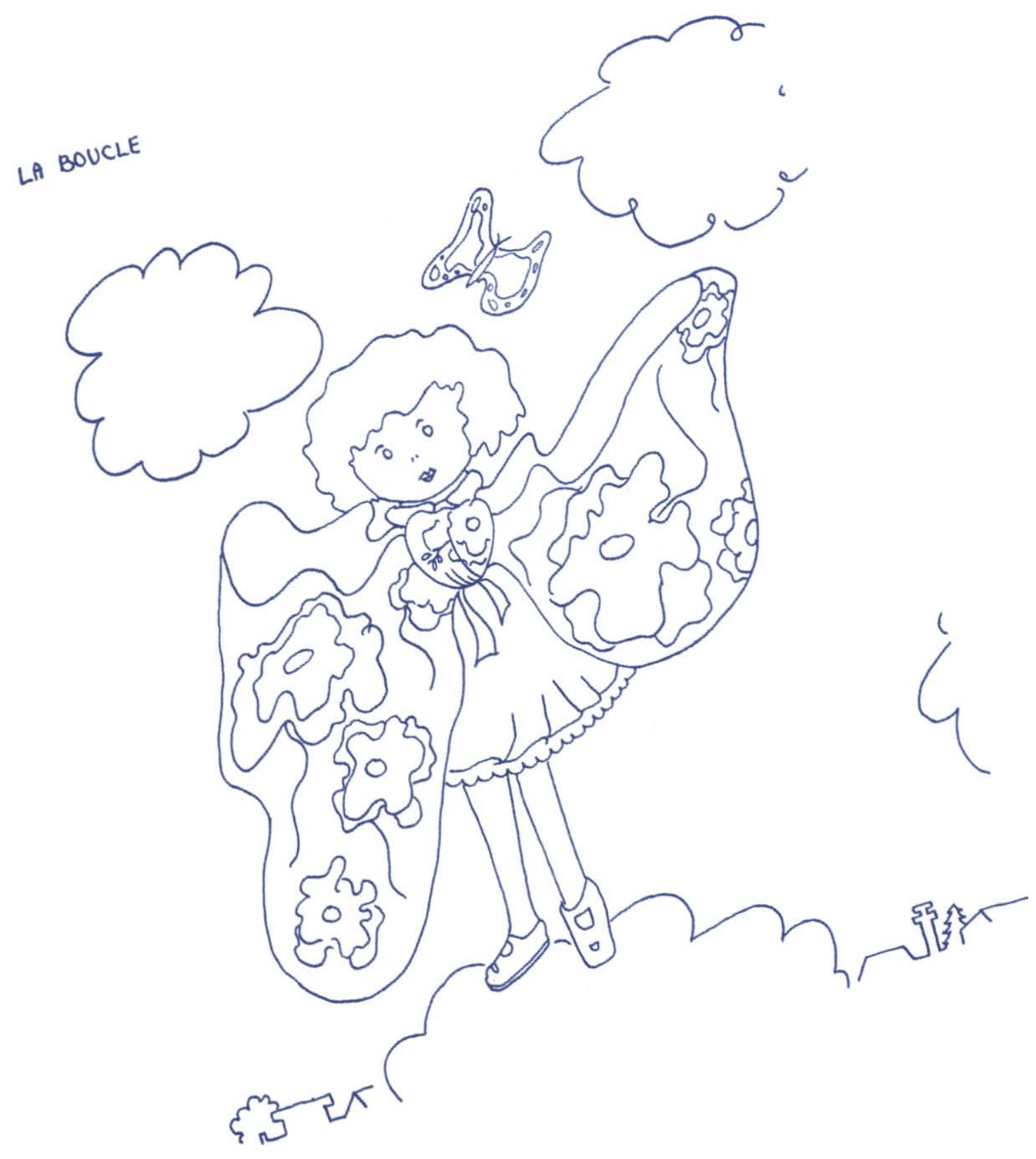


